\title{
Mesenteric cyst detection and segmentation by multiple K-means clustering and iterative Gaussian filtering
}

\author{
Mohamed Nasor, Walid Obaid \\ Department of Biomedical Engineering, Ajman University, United Arab Emirates
}

\begin{tabular}{l}
\hline \hline Article Info \\
\hline Article history: \\
Received Sep 17, 2020 \\
Revised May 12, 2021 \\
Accepted Jun 12, 2021 \\
\hline
\end{tabular}

\section{Keywords:}

Abdominal tumor

Automatic detection

CT scans

Iterative Gaussian filtering

Mesenteric cyst

\begin{abstract}
In this article a fully automated machine-vision technique for the detection and segmentation of mesenteric cysts in computed tomography (CT) images of the abdominal space is presented. The proposed technique involves clustering, filtering, morphological operations and evaluation processes to detect and segment mesenteric cysts in the abdomen regardless of their texture variation and location with respect to other surrounding abdominal organs. The technique is comprised of various processing phases, which include K-means clustering, iterative Gaussian filtering, and an evaluation of the segmented regions using area-normalized histograms and Euclidean distances. The technique was tested using 65 different abdominal CT scan images. The results showed that the technique was able to detect and segment mesenteric cysts and achieved 99.31\%, 98.44\%, 99.84\%, 98.86\% and $99.63 \%$ for precision, recall, specificity, dice score coefficient and accuracy respectively as quantitative performance measures which indicate very high segmentation accuracy.
\end{abstract}

This is an open access article under the CC BY-SA license.

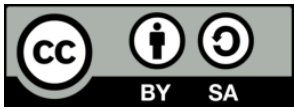

\section{Corresponding Author: \\ Mohamed Nasor \\ Department of Biomedical Engineering \\ Ajman University \\ Ajman, United Arab Emirates \\ Email: m.nasor@ajman.ac.ae}

\section{INTRODUCTION}

Mesenteric cysts are mostly, benign intra-abdominal tumors with probability of one occurrence in every 250,000 hospital admission cases [1], while malignant mesenteric cysts represent less than $3 \%$ of cases [2]. Mesenteric cysts are usually located in the mesentery but they may spread to the retro-peritoneum. They can appear in any part within the mesentery of the gastrointestinal tract from duodenum to rectum. The majority of cysts occur in the small bowl mesentery, compared to few occurrences in the large bowl mesentery and retroperitoneum [3]. The sizes of mesenteric cysts vary from very small in the range of millimeters to very large mimicking tubercular ascites [4]. The cause of these cysts are not yet known but there are many theories about their development [5]. The pathological symptoms of the development of mesenteric cysts are non-specific; they can cause pain in $82 \%$ of cases; nausea and vomiting in $45 \%$ of cases; constipation in $27 \%$ of cases; and diarrhea in $6 \%$ of cases. They can also cause other complications such as spillage of infective fluid, volvulus, and obstruction [6]. Most of these cysts are discovered accidentally when a patient is undergoing medical check-up for other health problems such as small-bowel obstruction, appendicitis, or diverticulitis. Also, in some patients, the cyst is diagnosed when it grows in size to the extent of causing visible enlargement of the abdomen causing discomfort and pain. The usual treatment of a mesenteric cyst is a complete excision in order to avoid recurrence and possible development to malignant transformations. Some cases may require bowel resection if the cyst is very close to bowel structures or 
contain blood vessels that are linked to the bowel. Since the only effective treatment of the mesenteric cysts is surgical operation, early noninvasive detection and localization of these cysts may prevent further complications associated with surgical excision when they become large. Various automated segmentation techniques for detecting specific abnormalities and lesions in the abdomen using CT scan images were discussed in the literature. A technique that is based on the texture analysis of the region of interest within the image was presented by Heimann et al [7]. Graph cuts that use shape enhancement constraints combined with level set techniques were used for CT scan image segmentation by incorporating likelihood energy to approximate the density distribution of the target area and background [8]. Various techniques that use Sigmoid edge modeling and machine learning that combine SVM, watershed, and scattered data approximation algorithms were presented by various researchers [9]. Nonparametric intensity distribution estimation and a hidden Markov measure field model were also proposed [10]. Deep convolutional neural networks (DCNN) techniques were proposed to achieve computer vision tasks such as image segmentation, classification, and recognition [11]. These techniques extract important features (weights and biases) convoluted with various objects in the image. Advanced forms of CNN segmentation based on fully convolutional neural networks FCN were proposed by Chen et al [12]. A combination of CNN and FCNs techniques were also suggested for medical image analysis [13].

\section{RESEARCH METHOD}

Figure 1 shows a block diagram of the proposed technique. The first step in the technique is applying K-means clustering using $\mathrm{N}$ clusters. K-means clustering aims to partition any data set $\mathrm{X}=\mathrm{x} 1, \ldots, \mathrm{xn}, \mathrm{xn} \in R^{d}$ into $\mathrm{M}$ subsets $\mathrm{C} 1, \ldots, \mathrm{Cn}$ such that the sum of the squared Euclidean distances are optimized between each data point xi and the centroid mk (cluster center) of the subset Ck that contains xi [14]. The clusters are determined by minimizing the sum of the errors given by (1):

$$
E\left(m 1, \ldots, m_{M}\right)=\sum_{i=1}^{N} \sum_{k=1}^{M} I\left(x_{i} \in C_{k}\right)\left|x_{i}-m_{k}\right|^{2}
$$

where $\mathrm{I}(\mathrm{xi})=1$ if $(\mathrm{xi} \in \mathrm{Ck})$, otherwise $\mathrm{I}(\mathrm{xi})=0$.
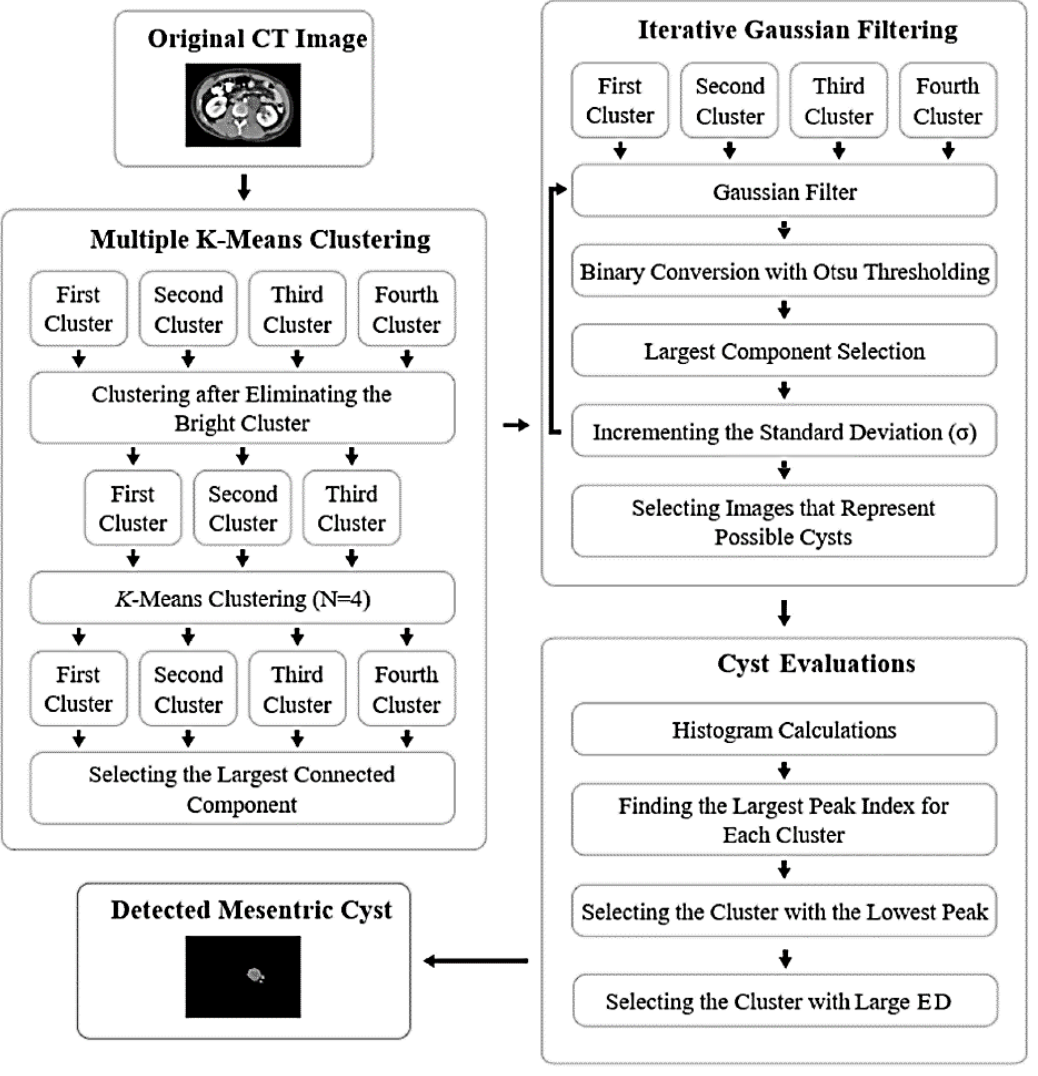

Figure 1. Block diagram of the proposed technique 
The purpose of the initial clustering is to eliminate the bright regions that most likely do not represent the cyst. Eliminating the bright regions is done based on sorting the $\mathrm{N}$ clusters by the histogram peak index value and selecting the cluster that has the highest histogram peak index. K-means clustering is applied again using $\mathrm{N}$ clusters in order to exclude the false regions by morphologically selecting the largest region from each cluster. $\mathrm{N}$ was set to 4 to produce 4 clusters which include a dark cluster, a medium-dark cluster, a medium-bright cluster, and a bright cluster. The value of $\mathrm{N}$ was set to 4 because mesenteric cysts have different average intensity values than other surrounding regions such as the liver and in order to expand the segmented regions of the cysts by increasing the number of true positive pixels since the cyst area was found to shrink when $\mathrm{N}$ becomes greater than 4. Table 1 and Figure 2 show an example of using different values of $\mathrm{N}$ on three different $\mathrm{CT}$ scan images. It can be seen that the maximum number of segmented true positive pixels are obtained when $\mathrm{N}$ is 4 . Since the cyst usually occurs in darker clusters, the bright cluster is eliminated from the image. K-means clustering is applied again using $\mathrm{N}=4$ followed by Gaussian filtering on the four clusters iteratively while increasing the square root of the variance $(\sigma)$ in each iteration, starting from $\sigma=1$ until there is no significant change is noticed when the value of $\sigma$ increases as shown in the example in Figure 3. Increasing the filter variance will smooth out noise and eliminate all edges including phantom edges [15]. The two-dimensional iterative Gaussian filter used is described by (2):

$$
G(x, y, n)=\frac{1}{2 \pi\left(\sigma_{n}^{2}\right)} e^{\frac{-\left(x^{2}+y^{2}\right)}{2\left(\sigma_{n}\right)^{2}}}, \quad n=1,2,3, \ldots
$$

where $\sigma \mathrm{n}$ is the variance of the Gaussian filter at the $\mathrm{n}$ th iteration.

Table 1. Number of true positive pixels for different values of $\mathrm{N}$ (number of clusters)

\begin{tabular}{ccccc}
\hline & 4 clusters & 5 clusters & 6 clusters & 7 clusters \\
\hline Case 1 & 88251 & 88082 & 86086 & 85166 \\
Case 2 & 29727 & 27933 & 27398 & 26288 \\
Case 3 & 5621 & 5364 & 5311 & 4904 \\
\hline
\end{tabular}

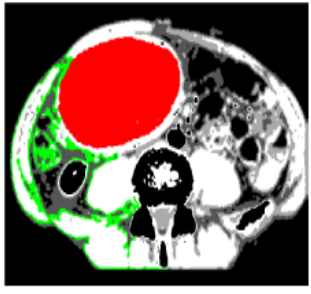

(a)

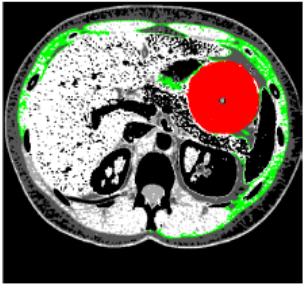

(e)

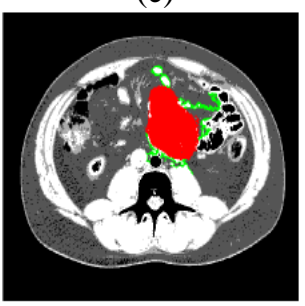

(i)

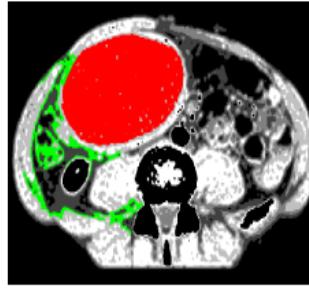

(b)

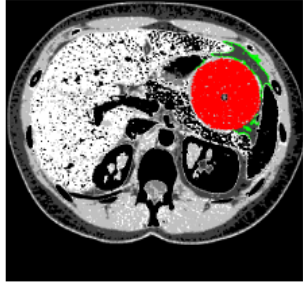

(f)

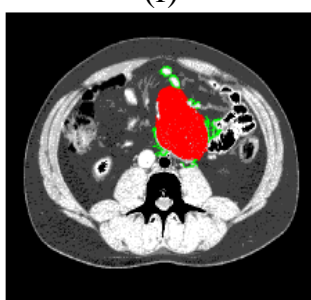

(j)

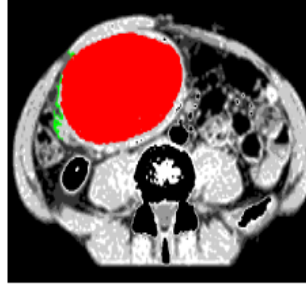

(c)

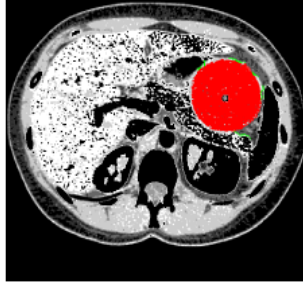

(g)

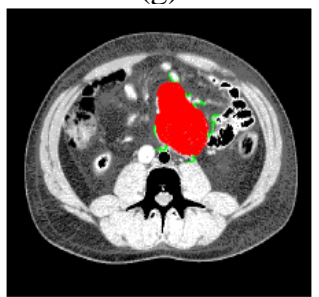

(k)

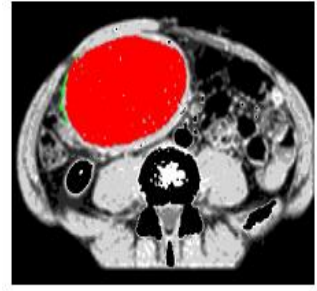

(d)

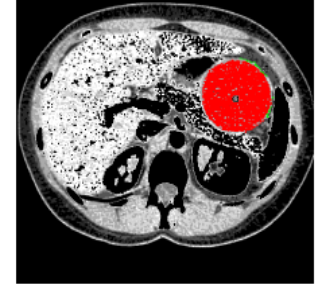

(h)

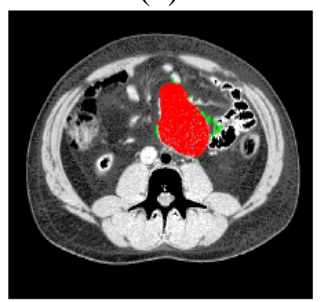

(1)

Figure 2. Applying K-means clustering using different values of $\mathrm{N}$, (a)-(d) varying the value of $\mathrm{N}$ between 4 and 7 for the first image, (e)-(h) varying the value of $\mathrm{N}$ between 4 and 7 for the second image,

(i)-(1) varying the value of $\mathrm{N}$ between 4 and 7 for the third image 
The false surrounding edges that are attached to each of the possible regions of the cysts are also removed by converting the image into binary image and performing iterative Gaussian filtering process using Otsu's threshold value [16]. The largest region that has the smallest number of non-zero elements is selected for further processing. In order to maximize the removal of false surrounding edges, the Gaussian iterative process and binary conversion were repeated on the resultant largest regions using small threshold value rather than Otsu's in order to focus on the central significant regions. In order to expand the region of the possible cyst area, region growing was applied using the center index point as a seed point [17]. The validity of the mesenteric cyst assessment is determined by finding the area normalized histograms and the euclidean distance values. Area normalized histograms are obtained for the resultant medium-bright and medium-dark regions. The region with a histogram that has the shortest dominant peak index is selected as the cyst. In addition, the euclidean distance of the cyst center index with respect to the horizontal bottom line of the image is also calculated. The region is not considered to be a cyst if the value of the euclidean distance is very small.

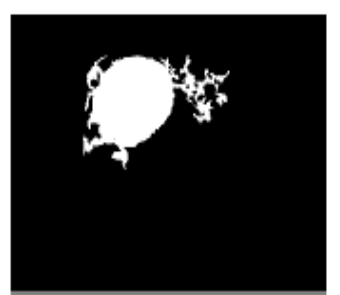

(a)

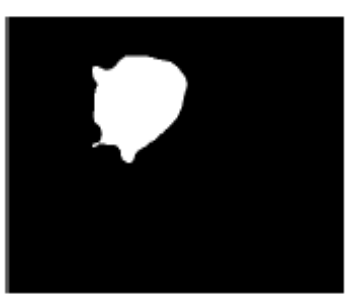

(b)

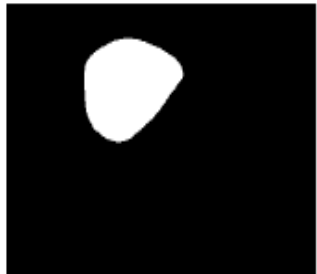

(d)

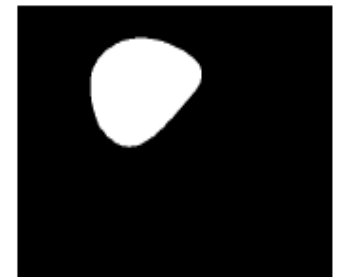

(e)

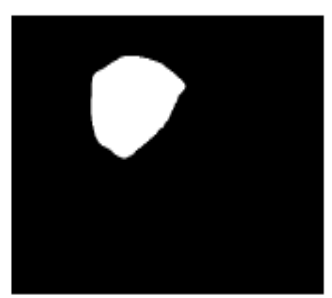

(c)

Figure 3. The effect of increasing the value of $\sigma$ in the iterative Gaussian filtering process

Figure 4(a) shows a CT scan image that contains a mesenteric cyst. The first K-means clustering of the image is shown in Figure 4(b). Figure 4(c) shows the result of the second K-means clustering after eliminating the bright cluster in (a). It can be noticed that the bright regions in the original image were completely eliminated as shown in Figures 4(d)-4(g). Figures 4(f)-4(g) contain the internal regions as compared with Figures 4(d)-4(e). Iterative Gaussian filter was applied afterwards on all the four clusters using both Otsu and small threshold values. This process eliminated the surrounding edges which were attached to the cyst and other false regions as shown in Figures 4(h)-4(k). Figure 5 shows the process of evaluating the selected medium-dark and medium-bright regions (after applying region growing) based on finding the area-normalized histograms and comparing the peak value indices of 5 different CT images. It can be noticed for each case, that the true cyst has a lower dominant histogram peak index compared to the dominant histogram index of false region as shown in Table 2. Figures 6 and 7 show the process of evaluating cyst regions by finding the Euclidean distance value with respect to the horizontal bottom line for eight different cases. The regions are discarded in the first four cases because of the small Euclidean distance values while the cysts in the remaining four cases are selected.

Table 2. Histogram peak evaluation for five mesenteric cysts

\begin{tabular}{cccc}
\hline $\begin{array}{c}\text { CT Scan } \\
\text { Image }\end{array}$ & $\begin{array}{c}\text { True Mesenteric Cyst } \\
\text { Histogram Peak Index }\end{array}$ & $\begin{array}{c}\text { False Mesenteric Cyst } \\
\text { Histogram Peak Index }\end{array}$ & Difference \\
\hline Image 1 & 106 & 138 & 32 \\
Image 2 & 105 & 129 & 24 \\
Image 3 & 104 & 127 & 23 \\
Image 4 & 99 & 126 & 27 \\
Image 5 & 126 & 163 & 37 \\
\hline
\end{tabular}




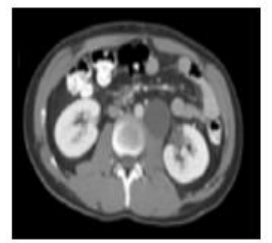

(a)

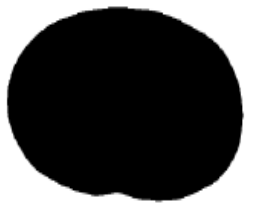

(e)

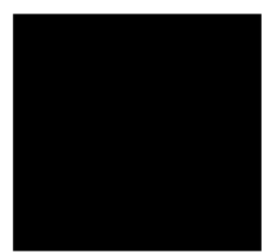

(i)

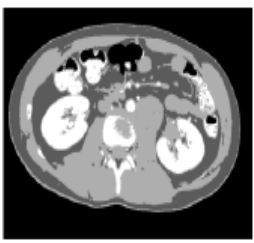

(b)

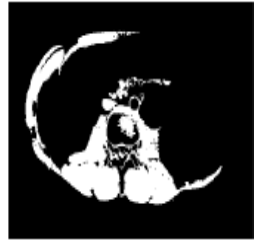

(f)

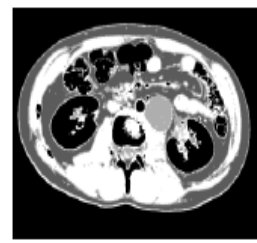

(c)

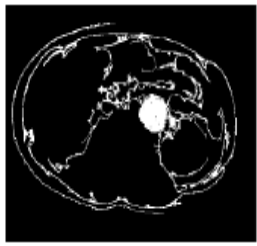

(g)

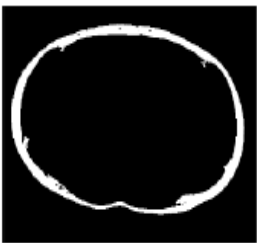

(d)

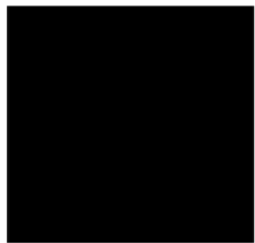

(h)

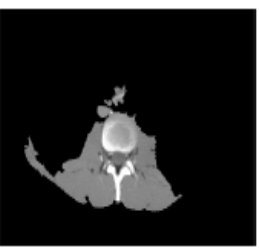

(j)

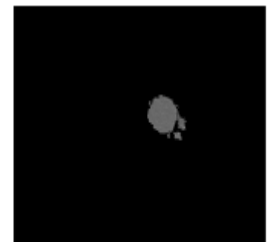

(k)

Figure 4. Applying K-means clustering and iterative Gaussian filtering on a CT scan image, (a) original CT image, (b) applying K-means clustering on the original CT scan image, (c) applying K-means clustering on the CT scan image after eliminating the bright cluster from the first clustered image, $(\mathrm{d})-(\mathrm{g})$ four clusters of the CT image after eliminating the bright cluster (medium-dark, dark, bright, and medium bright), (h)-(k) result of applying iterative Gaussian filtering on each of the four clusters shown in (d)-(g) respectively

\begin{tabular}{|c|c|c|c|c|}
\hline \multirow[b]{2}{*}{ CT Image \# } & \multicolumn{2}{|c|}{ True Cyst } & \multicolumn{2}{|c|}{ False Cyst } \\
\hline & $\begin{array}{c}\text { True Mesenteric } \\
\text { Cyst }\end{array}$ & True Histogram & $\begin{array}{c}\text { False } \\
\text { Mesenteric Cyst }\end{array}$ & False Histogram \\
\hline Image 1 & & 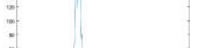 & & 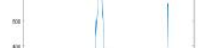 \\
\hline Image 2 & & $=$ & & 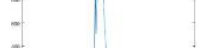 \\
\hline Image 3 & & $=$ & & \\
\hline Image 4 & & $=$ & & $=$ \\
\hline Image 5 & & & & \\
\hline
\end{tabular}

Figure 5. Histogram evaluation of the resultant segmented regions of interest for 5 cases. The true mesenteric cysts have peak indeces around 100, while false mesenteric regions have indeces much higher than the peak of their corresponding true mesenteric cyst histograms 


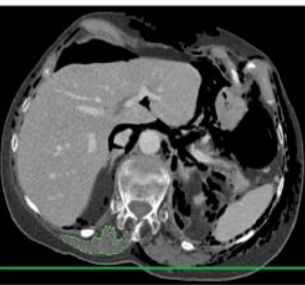

(a)

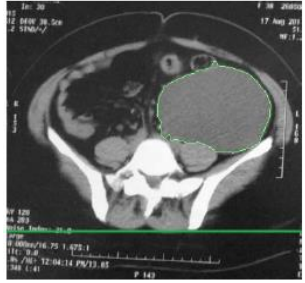

(e)

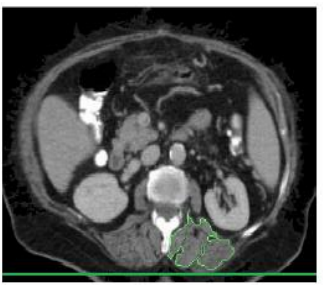

(b)

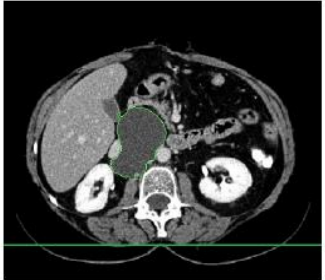

(f)

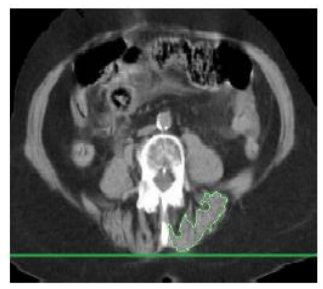

(c)

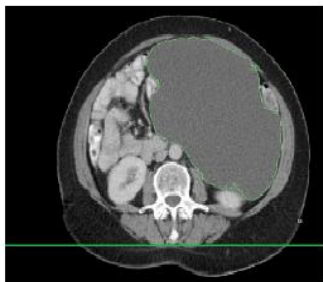

(g)

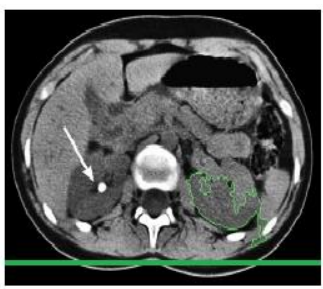

(d)

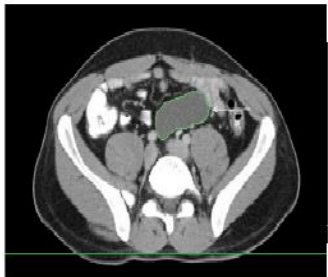

(h)

Figure 6. Evaluating the cysts by finding the Euclidean distance value; (a)-(d) segmented regions were not considered because of their proximity to the edges, (e)-(h) segmented regions were chosen as true cysts

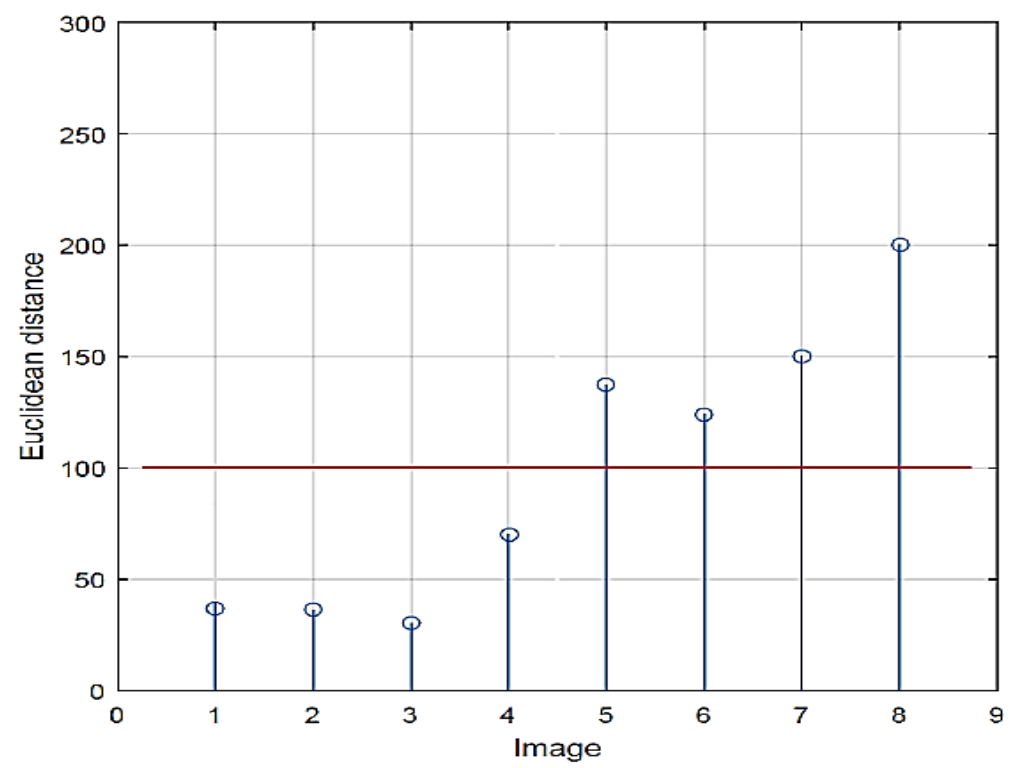

Figure 7. Euclidean distance evaluations of the cysts

\section{RESULTS AND DISCUSSION}

The proposed mesenteric cyst detection and segmentation technique was tested using a PC with Intel Core i5-7300U 2.7 GHz and 8 GB of RAM, the code was developed using MATLAB R2015a. The proposed technique was tested on 65 different CT scan abdominal images with different views: Transverse (head to toe) plane, coronal (front to back) plane, and sagittal (side-to-side) plane. The majority were transverse images while the remaining were coronal and sagittal images. Twelve images did not contain mesenteric cysts while 53 images contained mesenteric cysts with varying size, shape, location, and intensity values. The dataset of images was obtained from [18]-[21]. The novelty of the proposed technique is that it was able to detect and segment mesenteric cysts in all the CT scan images without the need to perform training of image datasets as in machine learning techniques. Moreover, the proposed technique was able to perform the detection and segmentation regardless of the different locations, shapes, sizes, and intensity values of the cysts. Figure 8 shows the detection and segmentation results after implementing the proposed technique. The technique was also tested on CT scan images that do not contain mesenteric cysts. Figure 9 shows the detected false regions in these CT images. These false regions were discarded based on their histogram peak indexes and Euclidean distance from the edges. 


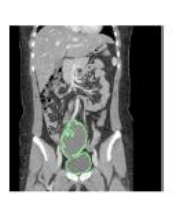

1
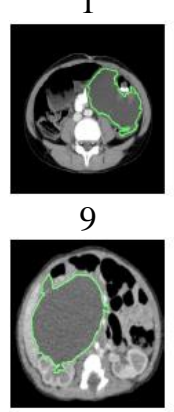

17

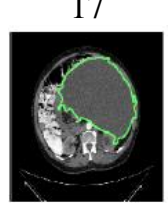

25

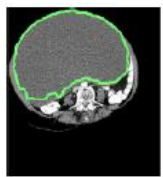

33

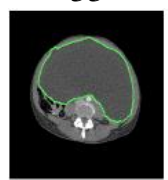

41

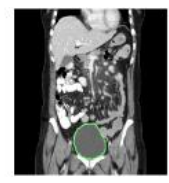

2

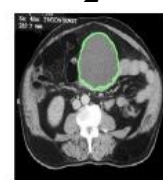

10

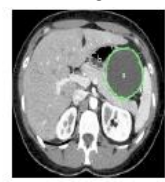

18

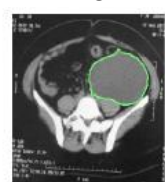

26

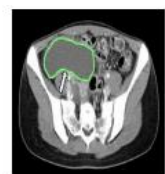

34

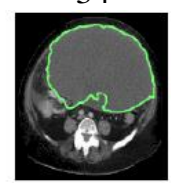

42

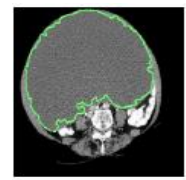

49
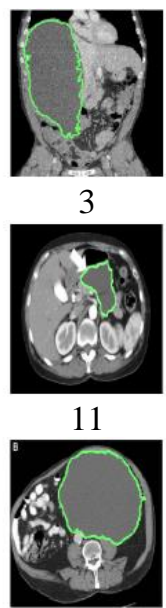

19

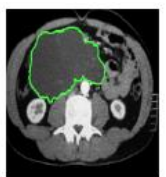

27

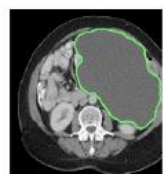

35

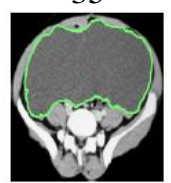

43

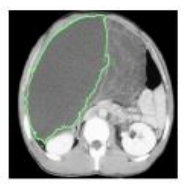

50

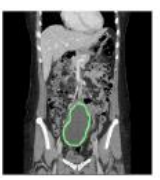

4

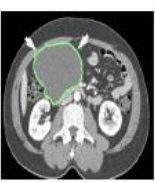

12

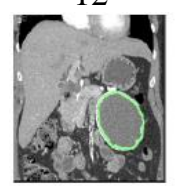

20

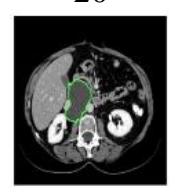

28

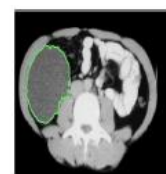

36

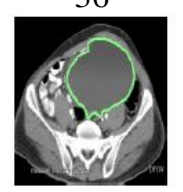

44

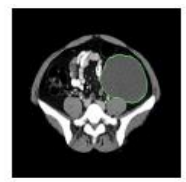

51
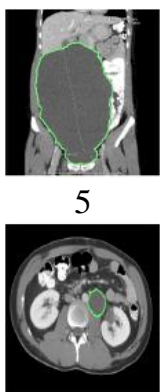

13

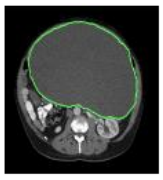

21

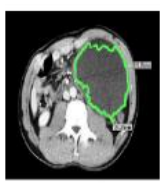

29

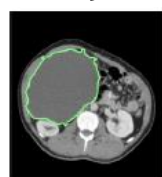

37

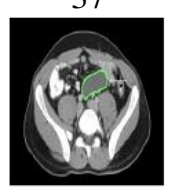

45

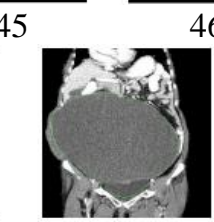

52

30
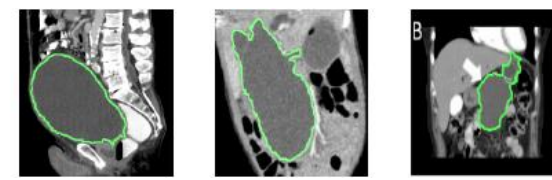

6
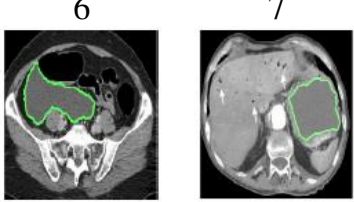

14

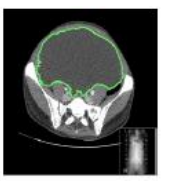

22
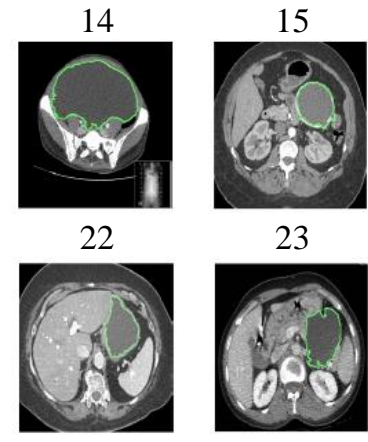

23

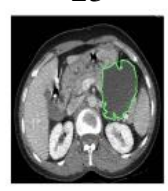

31
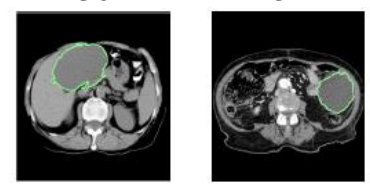

39
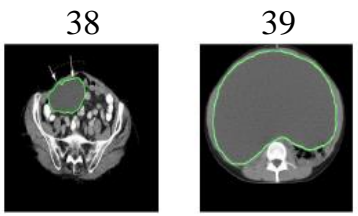

47

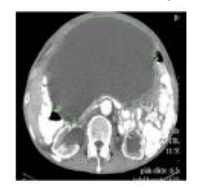

53
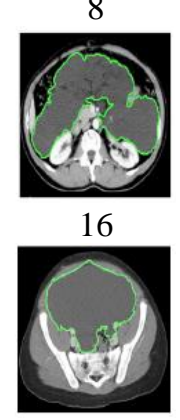

24

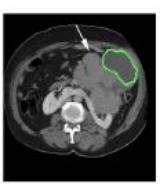

32

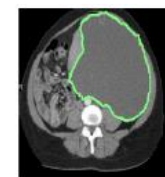

40

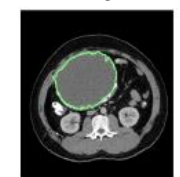

48

Figure 8. Mesenteric cyst segmentation and localization using the proposed technique in $53 \mathrm{CT}$ scan images that were obtained from [18]-[21]

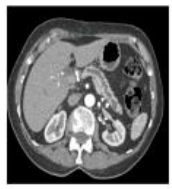

1

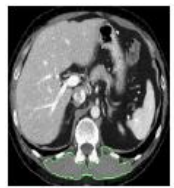

7

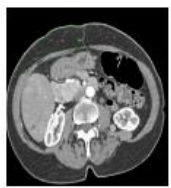

2

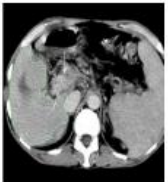

8

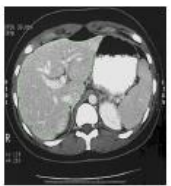

3

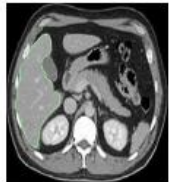

9

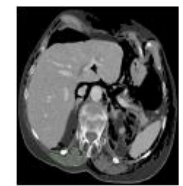

4

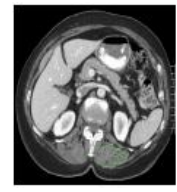

10

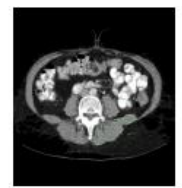

5

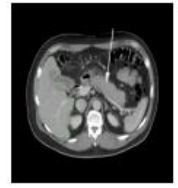

11

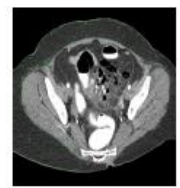

6

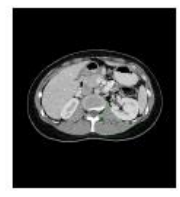

12

Figure 9. The detected false regions after applying the proposed technique on 12 different CT scan images without mesenteric cyst 
The effectiveness of the proposed technique was evaluated by calculating the performance measurement parameters for each of the segmented CT scan images. The performance measurement parameters used are precision, recall, specificity, dice score coefficient, and accuracy. The calculation of these parameters was based on the average values of the true positive (TP), false positive (FP), true negative $(\mathrm{TN})$, and false negative (FN) pixels. These four categories of pixels were counted for each CT scan image in order to calculate the performance measurement parameters using (3)-(7). Figure 10 shows the dispersion plot of the values for precision, recall, specificity, dice score coefficient (DSC), and accuracy. In the figure the box plots represent quartile ranges of the scores; whiskers and dots '.' indicate outliers; and crosses ' $x$ ' indicate the mean values. It can be noted that the mean value of precision is $99.31 \%$ while the mean value of Recall is $98.44 \%$. And the mean values of specificity, dice score coefficient and accuracy are $99.84 \%$ and $98.86 \%, 99.63 \%$ respectively. It can also be noticed that the proposed technique has high percentages for the performance parameters as the mean values of precision, specificity, and accuracy are all above $99.00 \%$ while the mean values of recall and dice score coefficient are above $98.00 \%$. The maximum and minimum values of the performance parameters are: $100.00 \%$ and $94.25 \%$ for precision, $100.00 \%$ and $93.63 \%$ for Recall, $100.00 \%$ and $99.09 \%$ for specificity, $99.99 \%$ and $96.75 \%$ for DSC, $99.99 \%$ and $98.30 \%$ for accuracy respectively. These values indicate that the performance of the proposed technique was satisfactory. The proposed technique can assist clinicians in diagnosing and treatment planning for patients with mesenteric cysts.

$$
\begin{aligned}
& \text { Precision }=\frac{T P}{T P+F P} \\
& \text { Recall }=\frac{T P}{T P+F N} \\
& \text { Specificity }=\frac{T N}{T N+F P} \\
& \text { DSC }=\frac{2 T P}{2 T P+F P+F N} \\
& \text { Accuracy }=\frac{T P+T N}{T P+F P+F N+T N}
\end{aligned}
$$

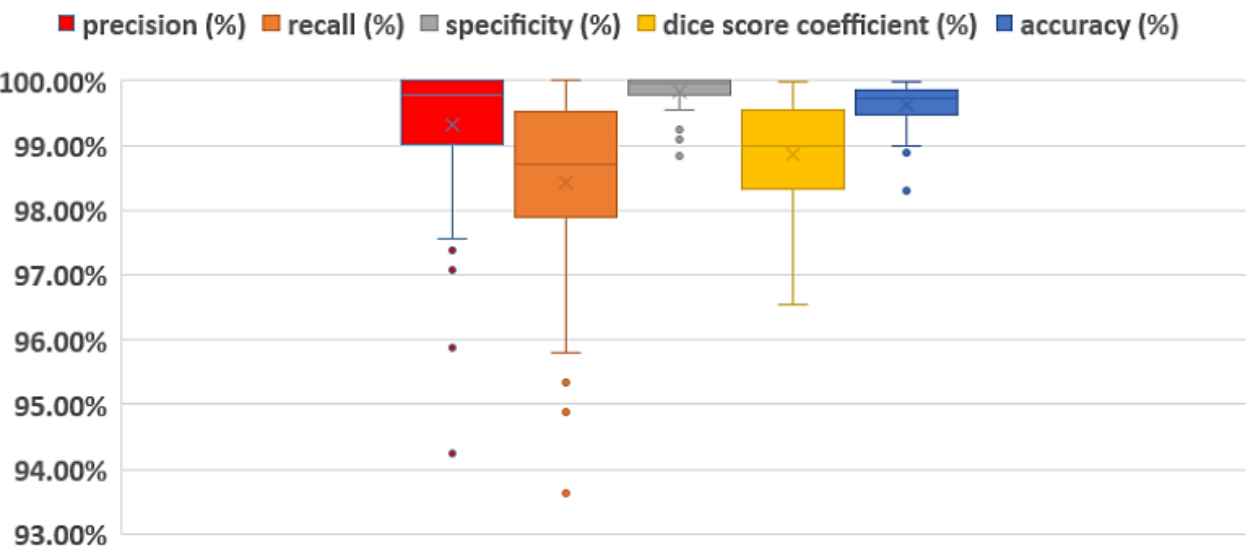

Figure 10. Dispersion of the performance parameters scores of the proposed technique (precision, recall, specificity, dice score coefficient, and accuracy)

The performance of the proposed technique was also compared with other CT scan cyst segmentation techniques. The comparison was based on the values for specificity, sensitivity, accuracy, and DSC as shown in Tables 3 and 4. It can be noticed that the precision and recall values of the proposed technique are $99.31 \%$ and $98.44 \%$ which are larger than the precision and recall values of both FCNN and contourlet techniques by more than $2.75 \%$. The Specificity value of the proposed technique is larger than the specificity value of the contourlet technique by more than $1 \%$. The DSC value of the proposed technique is $98.86 \%$ which is larger than the DSC values of the FCNN, Self-learning, and deep supervision techniques by more than $11 \%$. 
Table 3. Comparison between the performance of the proposed technique and other techniques

\begin{tabular}{cccc}
\hline Technique & Precision & Recall & Specificity \\
\hline FCNN [22] & $91 \%$ & $86 \%$ & - \\
Contourlet [23] & $95.45 \%$ & $95.45 \%$ & $98.59 \%$ \\
Proposed & $99.31 \%$ & $98.44 \%$ & $99.84 \%$ \\
\hline
\end{tabular}

Table 4. Comparison between the performance of the proposed technique and other techniques

\begin{tabular}{ccc}
\hline Technique & DSC & Accuracy \\
\hline FCNN [22] & $87 \%$ & - \\
Self-learning [24] & $82.65 \%$ & - \\
Deep supervision [25] & $63.44 \%$ & - \\
Proposed & $98.86 \%$ & $99.63 \%$ \\
\hline
\end{tabular}

\section{CONCLUSION}

In this article, a fully automated technique for detecting, segmenting, and identifying mesenteric cysts in CT scan images was developed and tested. The technique consists of K-means clustering, iterative Gaussian filtering, and an evaluation process using area-normalized histograms and Euclidean distances. The proposed technique was tested on 65 different CT scan images with different views that include transverse, coronal, and sagittal planes. The performance of the proposed technique was satisfactory based on the highperformance parameters achieved for precision $(99.31 \%)$, recall $(98.44 \%)$, specificity $(99.84 \%)$, dice score coefficient $(98.86 \%)$, and accuracy $(99.63 \%)$. The technique does not require intervention from a user or training using datasets as in artificial neural networks and machine learning algorithms. In addition, the technique performs effectively under different conditions such as different cyst intensities, locations, and sizes. The future extension of this work will focus on the segmentation of multiple lesions and cysts in CT images.

\section{REFERENCES}

[1] S. Miliaras, S. Trygonis, A. Papandoniou, S. Kalamaras, C. Trygonis, and D. Kiskinis, "Mesenteric cyst of the descending colon: report of a case," Acta Chirurgica Belgica, vol. 106, no. 6, pp. 714-716, 2006, doi: 10.1080/00015458.2006.11679990.

[2] R. Kurtz, T. M. Heiman, J. Holt, and A. R. Beck, "Mesenteric and retroperitoneal cysts," Annals of Surgery, vol. 203, no. 1, pp. 109-112, 1986, doi: 10.1097/00000658-198601000-00017.

[3] M. Saviano et al., "Mesenteric cystic neoformations: report of two cases," Surgery Today, vol. 29, no. 2, pp. 174-177, 1999, doi: 10.1007/BF02482245.

[4] D. Zamir, M. Yuchtman, M. Amar, U. Shoemo, and P. Weiner, "Giant mesenteric cyst mimicking ascites," Harefuah, vol. 130, no. 10, pp. 683-684, 1996.

[5] R. Richard, "Mesenteric and omental cysts," Mosby Elsevier, 6th edn., 2006.

[6] M. Hassan, N. Dobrilovic, and J. Korelitz, "Large gastric mesenteric cyst: case report and literature review," The American Surgeon, vol. 71, pp. 571-573, 2005, doi: 10.1177/000313480507100706.

[7] T. Heimann et al., "Comparison and evaluation of methods for liver segmentation from CT datasets," IEEE Transactions on Medical Imaging, vol. 28, no. 8, pp. 1251-1265, 2009, doi: 10.1109/TMI.2009.2013851.

[8] G. Li, X. Chen, F. Shi, W. Zhu, J. Tian, and D. Xiang, "Automatic liver segmentation based on shape constraints and deformable graph cut in CT images," IEEE Transactions on Image Processing, vol. 24, no. 12, pp. 5315-5329, 2015, doi: 10.1109/TIP.2015.2481326.

[9] A. H. Foruzan and Y. W. Chen, "Improved segmentation of low-contrast lesions using sigmoid edge model," International Journal of Computer Assisted Radiology and Surgery, vol. 11, pp. 1276-1283, 2016, doi: 10.1007/s11548-015-1323-x.

[10] A. Ben-Cohen, E. Klang, I. Diamant, N. Rozendorn, M. M. Amitai, and H. Greenspan, "Automated method for detection and segmentation of liver metastatic lesions in follow-up CT examinations," Journal of Medical Imaging, vol. 2, no. 3, 2015, doi: 10.1117/1.JMI.2.3.034502.

[11] J. Long, E. Shelhamer, and T. Darrell, "Fully convolutional networks for semantic segmentation," Proceedings of the IEEE Conference on Computer Vision and Pattern Recognition, 2015, pp. 3431-3440, doi: 10.1117/1.JMI.2.3.034502.

[12] S. Zheng et al., "Conditional random fields as recurrent neural networks," Proceedings of the IEEE International Conference on Computer Vision, 2015, pp. 1529-1537, doi: 10.1109/ICCV.2015.179.

[13] K. Kamnitsas et al., "Efficient multi-scale 3D CNN with fully connected CRF for accurate brain lesion segmentation," Medical Image Analysis, vol. 36, pp. 61-78, 2017, doi: 10.1016/j.media.2016.10.004.

[14] S. Chakraborty, D. Paul, S. Das, and J. Xu, "Entropy weighted power k-means clustering," International Conference on Artificial Intelligence and Statistics, vol. 108, pp. 691-701, 2020. 
[15] J. J. Hwang and K. H. Rhee, "Gaussian filtering detection based on features of residuals in image forensics," 2016 IEEE RIVF International Conference on Computing and Communication Technologies, Research, Innovation, and Vision for the Future (RIVF), 2016, pp. 153-157, doi: 10.1109/RIVF.2016.7800286.

[16] J. Zhang and J. Hu, "Image segmentation based on 2D Otsu method with histogram analysis," Proceedings of 2008 International Conference on Computer Science and Software Engineering, 2008, vol. 6, pp. 105-108, doi: 10.1109/CSSE.2008.206.

[17] R. Rouhi, M. Jafari, S. Kasaei, and P. Keshavarzian, "Benign and malignant breast tumors classification based on region growing and CNN segmentation," Expert Systems with Applications, vol. 42, no. 3, pp. 990-1002, 2015, doi: 10.1016/j.eswa.2014.09.020.

[18] S. Sobnach et al., "Primary seromucinous cystadenocarcinoma of the mesentery," South African Journal of Surgery, vol. 53, no. 3, pp. 44-45, 2016.

[19] T. Barani et al., "A giant mucinous borderline neoplasm of the mesentery misdiagnosed as an adnexal cyst - a case report and literature review," Clinical Obstetrics, Gynecology and Reproductive Medicine, vol. 3, no. 1, pp. 1-4, 2017, doi: 10.15761/COGRM.1000171.

[20] A. Abdelaal et al., "Laparoscopy as a Diagnostic and Definitive Therapeutic Tool in Cases of Inflamed Simple Lymphatic Cysts of the Mesentery," Case Reports in Surgery, vol. 2015, 2015, doi: 10.1155/2015/325939.

[21] S. R. Challa, D. Senapati, T. K. Nulukurthi, and J. Chinamilli, "Mucinous mesenteric cyst of the sigmoid mesocolon: a rare entity," BMJ Case Reports, 2016, doi: 10.1136/bcr-2015-210411.

[22] N. Blau, E. Klang, N. Kiryati, M. Amitai, O. Portnoy, and A. Mayer, "Fully automatic detection of renal cysts in abdominal CT scans," International Journal of Computer Assisted Radiology and Surgery, vol. 13, no. 7, pp. 957-966, 2018, doi: 10.1007/s11548-018-1726-6.

[23] F. Abdolali, R. A. Zoroofi, Y. Otake, and Y. Sato, "Automated classification of maxillofacial cysts in cone beam CT images using contourlet transformation and Spherical Harmonics," Computer Methods and Programs in Biomedicine, vol. 139, pp. 197-207, 2017, doi: 10.1016/j.cmpb.2016.10.024.

[24] L. Zhang, V. Gopalakrishnan, L. Lu, R. M. Summers, J. Moss, and J. Yao, "Self-learning to detect and segment cysts in lung CT images without manual annotation," IEEE 15th International Symposium on Biomedical Imaging, 2018, pp. 1100-1103, doi: 10.1109/ISBI.2018.8363763.

[25] Y. Zhou, L. Xie, E. K. Fishman, and A. L. Yuille, "Deep supervision for pancreatic cyst segmentation in abdominal CT scans," International Conference on Medical Image Computing and Computer-assisted Intervention, 2017, pp. 222-230, doi: 10.1007/978-3-319-66179-7_26. 